\title{
Effect of Body Position on High-resolution Esophageal Manometry Variables and Final Manometric Diagnosis
}

\author{
Carlo G Riva, Stefano Siboni, Davide Ferrari, Marco Sozzi, Matteo Capuzzo, Emanuele Asti, Cristina Ogliari, and Luigi Bonavina* \\ Division of General and Foregut Surgery, Department of Biomedical Sciences for Health, University of Milano, IRCCS Policlinico San Donato \\ Milanese, Milano, Italy
}

\section{Background/Aims}

According to the Chicago classification version 3.0, high-resolution manometry (HRM) should be performed in the supine position. However, with the patient in the upright/sitting position, the test could more closely simulate real-life behavior and may be better tolerated. We performed a systematic review of the literature to search whether the manometric variables and the final diagnosis are affected by positional changes.

\section{Methods}

A literature search was conducted according to the Preferred Reporting Items for Systematic Reviews and Meta-analyses (PRISMA) statement. Studies published in English that compared HRM results in different body positions were included. Moreover, the change in diagnosis of esophageal motility disorders according to the shift of body position was investigated.

\section{Results}

Seventeen studies including 1714 patients and healthy volunteers met the inclusion criteria. Six studies showed a significant increase in lower esophageal sphincter basal pressure in the supine position. Integrated relaxation pressure was significantly higher in the supine position in 10 of 13 studies. Distal contractile index was higher in the supine position in 9 out of 10 studies. One hundred and fifty-one patients (16.4\%) out of 922 with normal HRM in the supine position were diagnosed with ineffective esophageal motility (IEM) when the test was performed in the upright position $(P<0.001)$.

\section{Conclusions}

Performing HRM in the upright position affects some variables and may change the final manometric diagnosis. Further studies to determine the normal values in the sitting position are needed.

(J Neurogastroenterol Motil 2020;26:335-343)

\section{Key Words}

Chicago; Esophageal motility disorders; Manometry; Reference values; Sitting position

Received: January 20, 2020 Revised: None Accepted: May 10, 2020

(a) This is an Open Access article distributed under the terms of the Creative Commons Attribution Non-Commercial License (http://creativecommons. org/licenses/by-nc/4.0) which permits unrestricted non-commercial use, distribution, and reproduction in any medium, provided the original work is properly cited.

${ }^{*}$ Correspondence: Luigi Bonavina, MD IRCCS Policlinico San Donato, Via Morandi 30, 20097 San Donato Milanese, Milano, Italy Tel: +39-0252774840, Fax: +39-3472601004,E-mail: luigi.bonavina@unimi.it 


\section{Introduction}

High-resolution manometry (HRM) is considered the test of choice to evaluate esophageal motility disorders. The first sets of normal values were established in $2006 .^{1-3}$ The normative values (5th and 95th percentiles) were obtained in 75 healthy volunteers studied in the supine position with a solid-state manometric assembly with 36 circumferential sensors spaced at $1-\mathrm{cm}$ intervals and ten $5-\mathrm{mL}$ water swallows in each subject. A subsequent study performed on 400 patients $^{4}$ allowed a classification of esophageal motility disorders, namely the Chicago classification, that has been updated and has now reached the third version. ${ }^{5}$ Normative thresholds can vary according to the HRM software system, catheter outer-diameter, bolus consistency and volume, age, obesity, ethnicity, and body position. ${ }^{6}$ Cutoffs for abnormality established in the supine position may not be valid in the upright/sitting position. Historically also, the conventional water-perfused esophageal manometry was performed with the patient lying in the supine position, which allowed to test the peristaltic function without interference of gravity on bolus transit. ${ }^{7}$ However, swallowing in the upright position is more similar to real-life behavior, may be more tolerable for patients with swallowing difficulties, and may reduce cardiovascular artifacts on the HRM tracing. ${ }^{8}$ The aim of this study is to perform a systematic review on comparative studies testing the results obtained during HRM in supine and upright/sitting positions and to search whether HRM variables are influenced by body location and may change the final diagnosis.

\section{Methods}

We conducted a systematic review according to the Preferred Reporting Items for Systematic Reviews and Meta-analyses (PRISMA) statement. An extensive literature search was conducted by 5 independent authors (C.G.R., D.F., M.C., M.S., and S.S.) to identify all clinical reports dealing with results of HRM comparing the supine and upright positions. PubMed, Cochrane, Embase, and Scopus databases were queried using the following terms: "body position", "esophageal manometry", "high resolution manometry", "HRM", and every possible combination with AND/OR. The search was restricted to studies published in English and was completed by consulting the listed references of each article. Studies with conventional esophageal manometry, and those assessing solid swallows, or which focused on upper esophageal sphincter parameters, or performed during general anesthesia, were excluded. Disagreements among authors were resolved by consensus; if no agreement could be reached, the senior author (L.B.) made the decision. For each selected study, data extracted included first author name, year of publication, nation where the study was performed, number of subjects involved, and whether they were healthy volunteers or symptomatic gastroesophageal reflux disease patients. The following parameters were recorded: protocol characteristics (angle of supine and upright position, number of swallows per set, water amount per swallow expressed in $\mathrm{mL}$, catheter outerdiameter expressed in $\mathrm{mm}$, and software used for data elaboration); upper esophageal sphincter characteristics, including basal pressure and residual pressure; lower esophageal sphincter (LES) characteristics, including basal pressure, integrated relaxation pressure (IRP), total and intra-abdominal length; and esophageal body characteristics, including distal contractile integral (DCI), intrabolus pressure (IBP), distal latency, mean peristaltic pressure, contractile pattern with percentage of failed, weak and rapid swallows, large and small breaks, and double-peak swallow. Lastly we reported the percentage of effective swallows and the change in diagnosis of esophageal motility disorders according to the shift of body position.

The methodological quality of the studies was assessed based on the most critical factors that increase the risk of bias in this specific context. ${ }^{9}$ Statistical analysis was performed using the SPSS software version 23 (IBM, Armonk, NY, USA). The rate of patients with ineffective esophageal motility (IEM) in the supine versus upright position was compared using Chi-Square Test, and the statistical significance was established at less than the 0.05 level.

\section{Results}

Ninety publications were found applying the search criteria. Twenty-seven publications were duplicated and were removed. Sixty-three studies were examined and further screening revealed that only 17 articles met the inclusion criteria (Figure). All included studies were designed as case-series and had a low to moderate risk for bias based on a global assessment of methodological quality.

\section{High-resolution Manometry Protocols}

One thousand seven hundred fourteen patients were included, ${ }^{10-26}$ of whom 1284 were symptomatic and 430 asymptomatic individuals. The sequence of the position assumed during HRM was described in almost all studies. Some authors referred that the exam was started in the supine position and the subsequent series of swallows was performed in the sitting/upright position; ${ }^{12,14,15,18,19,24}$ other referred the opposite. ${ }^{16,20,22,23}$ Patients were randomly assigned 

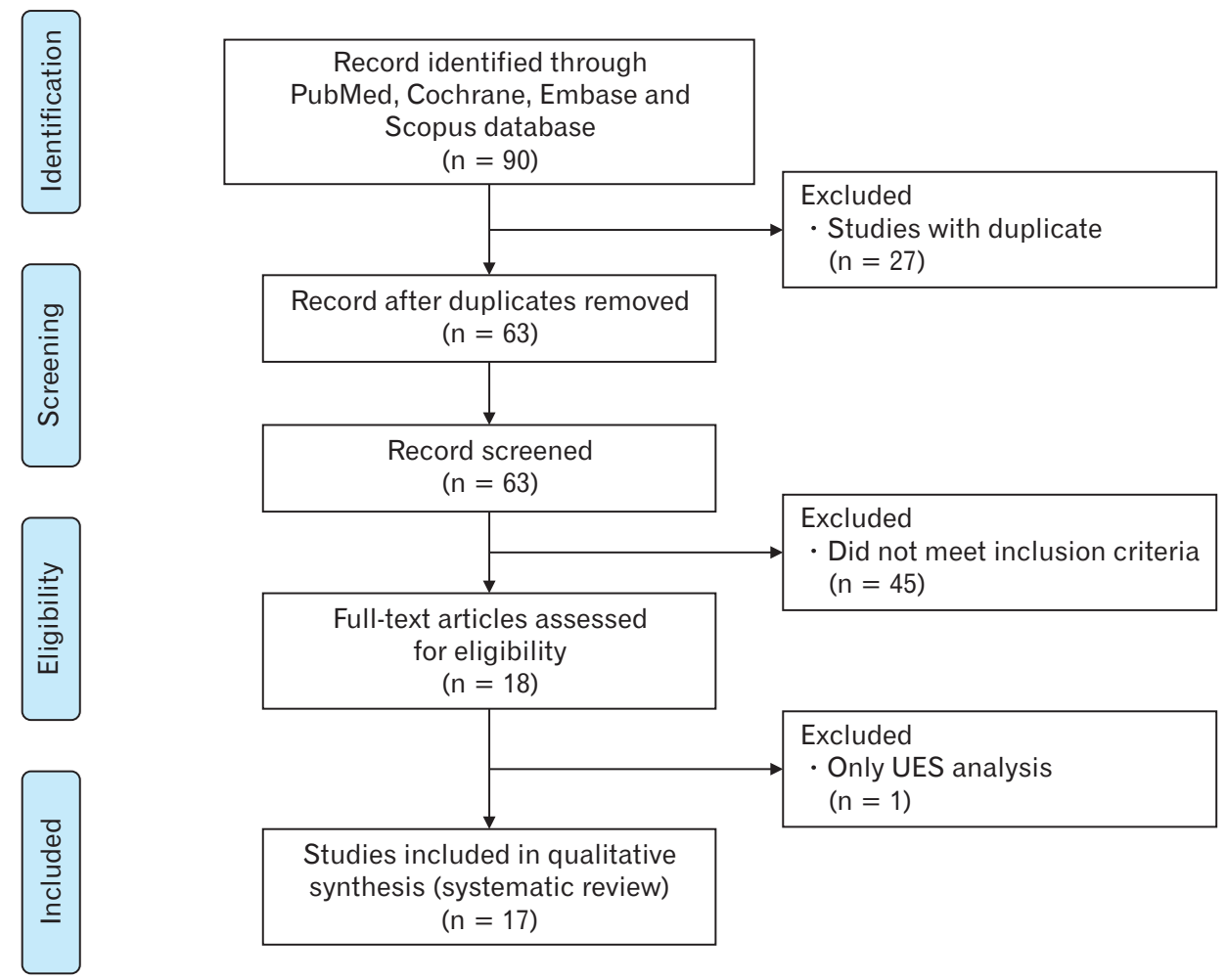

Figure. The Preferred Reporting Items for Systematic Reviews and Meta-analyses (PRISMA) diagram. UES, upper esophageal sphincter.

both to the upright or supine position in the studies by Zhang et $\mathrm{al}^{17,21}$ and Misselwitz et al. ${ }^{26}$ Only 5 studies ${ }^{14,16,17,19,24}$ reported the inclination assumed by the patient during the exam: between $0^{\circ}$ and $20^{\circ}$ for the supine position and between $75^{\circ}$ and $90^{\circ}$ for the upright/sitting position. Every study included at least 5 water bolus swallows per set for each position. Data were analyzed using various manometric softwares: 12 studies ${ }^{11-14,16,17,19-21,24-26}$ used Manoscan (of whom 3 and 9 from Medtronic [Minneapolis, MN, USA]), 3 studies ${ }^{15,22,23}$ used Bioview by Sandhill Scientific Inc (Ranch, CO, USA), and 2 studies ${ }^{10,18}$ used Trace! V1.2 videomanometry system (Hebbard System, Melbourne, Australia) (Table 1).

\section{High-resolution Manometry Variables}

In regard to LES parameters, LES length was analyzed in 5 studies, ${ }^{11,15,19,21,23} 3$ of which ${ }^{15,21,23}$ found a greater LES length in the supine position. Six ${ }^{15,20,21,23,24,26}$ of 8 studies ${ }^{11,12,15,20,21,23,24,26}$ showed a significant increase in LES basal pressure in the supine position, 3 studies ${ }^{11-13}$ did not find differences, and one ${ }^{19}$ found a significantly higher pressure in the upright position. IRP was significantly higher in the supine position in 10 of 13 studies, ${ }^{14-17,20-24,26}$ while in 2 studies ${ }^{13,18}$ it was significantly increased in the upright position.

In regard to esophageal body parameters, DCI was higher in the supine position in 9 of 10 studies. ${ }^{12,13,16-18,20,22-24,26}$ In the study by Besanko et al, ${ }^{18}$ DCI value was significantly higher in the upright position in the subgroup of older healthy patients. Six studies $^{11-13,15,18,20}$ investigated mean peristaltic wave pressure: it was significantly higher in the supine position in 2 studies, ${ }^{12,20}$ comparable in 3 studies, ${ }^{11,13,15}$ and significantly higher in the upright position in 1 study. ${ }^{18}$ IBP was significantly higher in the supine position in 3 studies, ${ }^{20,23,24}$ whilst it was comparable in the 2 positions in 1 study. ${ }^{13}$ Lastly, distal latency was investigated in 7 studies ${ }^{16,17,20-24,26}$ and was significantly higher in the supine position in 4 studies. ${ }^{16,17,20,24}$ The HRM values recorded in healthy subjects are summarized in Table 2.

\section{Concordance of Final Manometric Diagnosis}

Only 6 studies $^{10,12,14,20,24,26}$ for a total of 922 patients reported the difference in terms of final diagnosis in the 2 positions. One hundred and fifty-one patients (16.4\%) with normal HRM in the supine position were diagnosed with IEM when the test was performed in the upright position $(P<0.05)$. Variations in final diagnosis including normal motility, IEM, absent peristalsis, distal esophageal spasm, hypercontractile motility, esophagogastric junction (EGJ) outflow obstruction, and achalasia are reported in Table 3. 


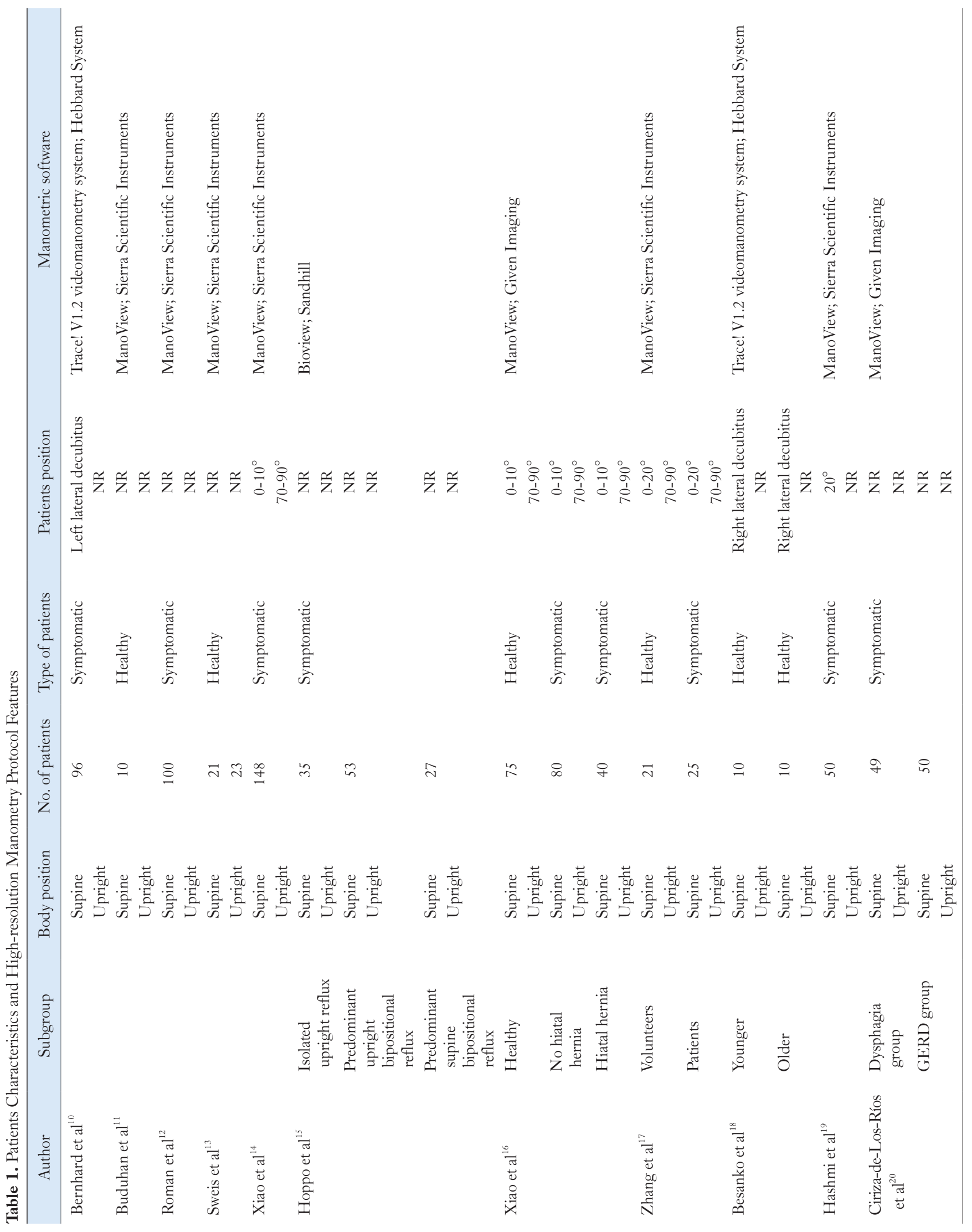




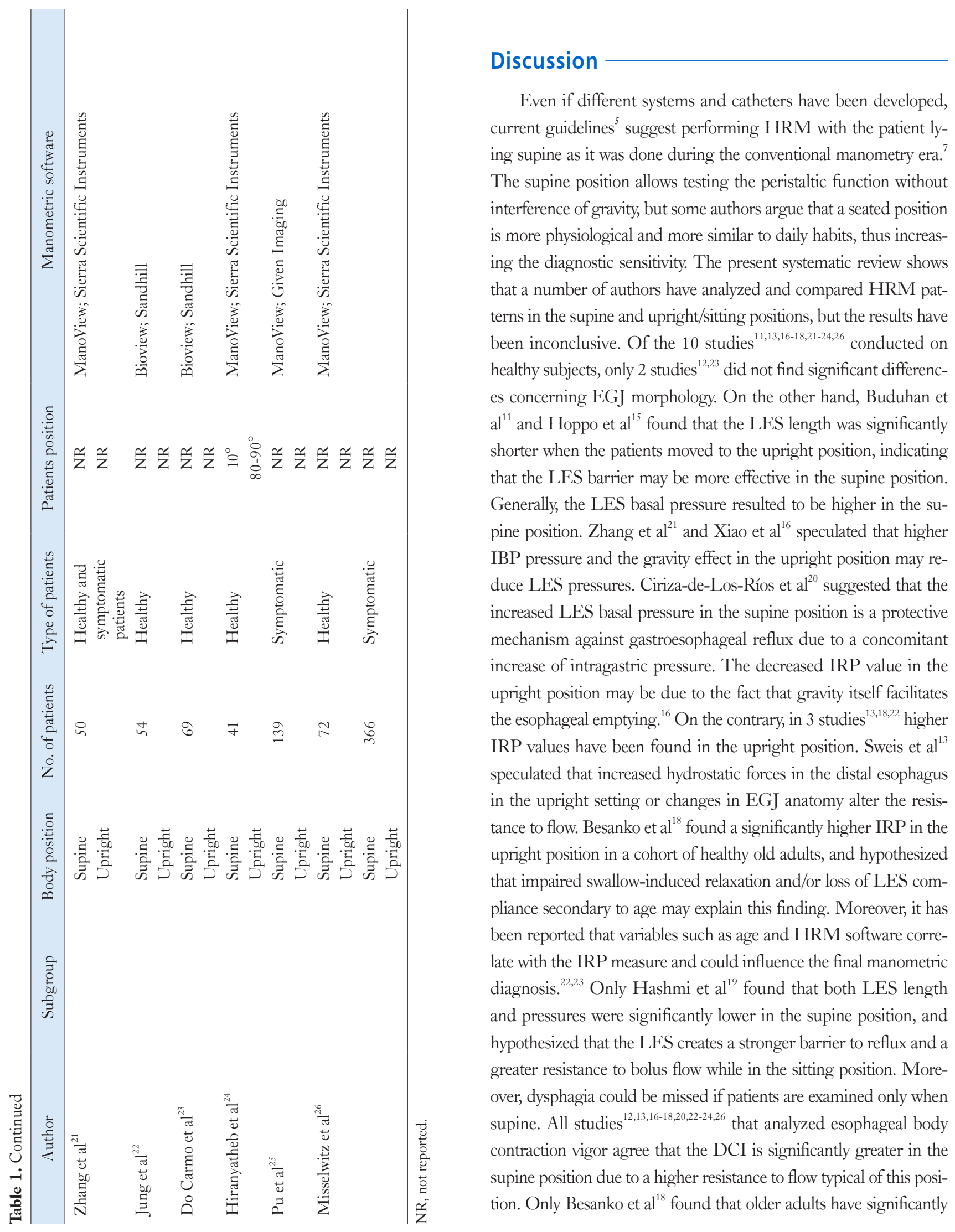




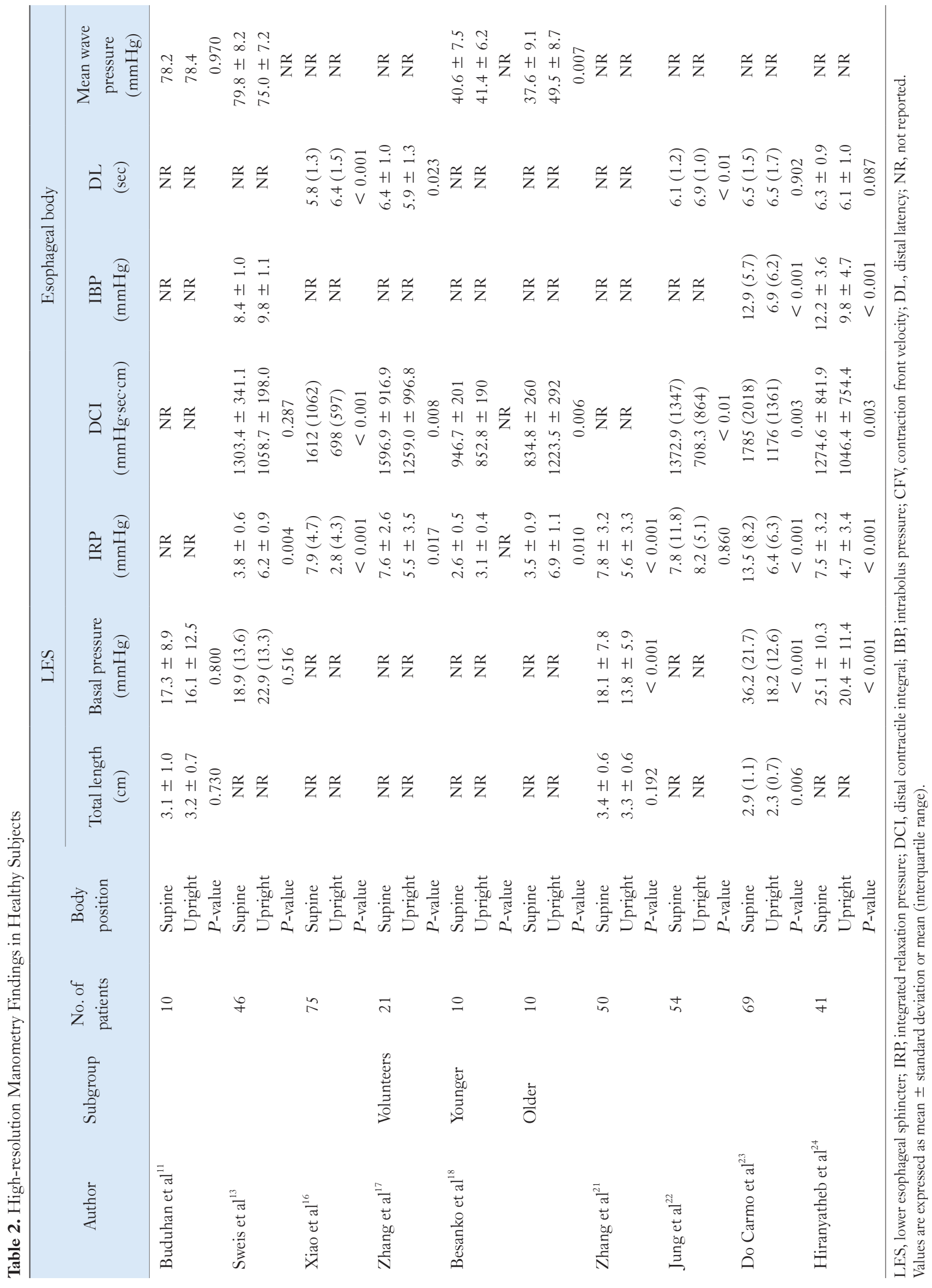




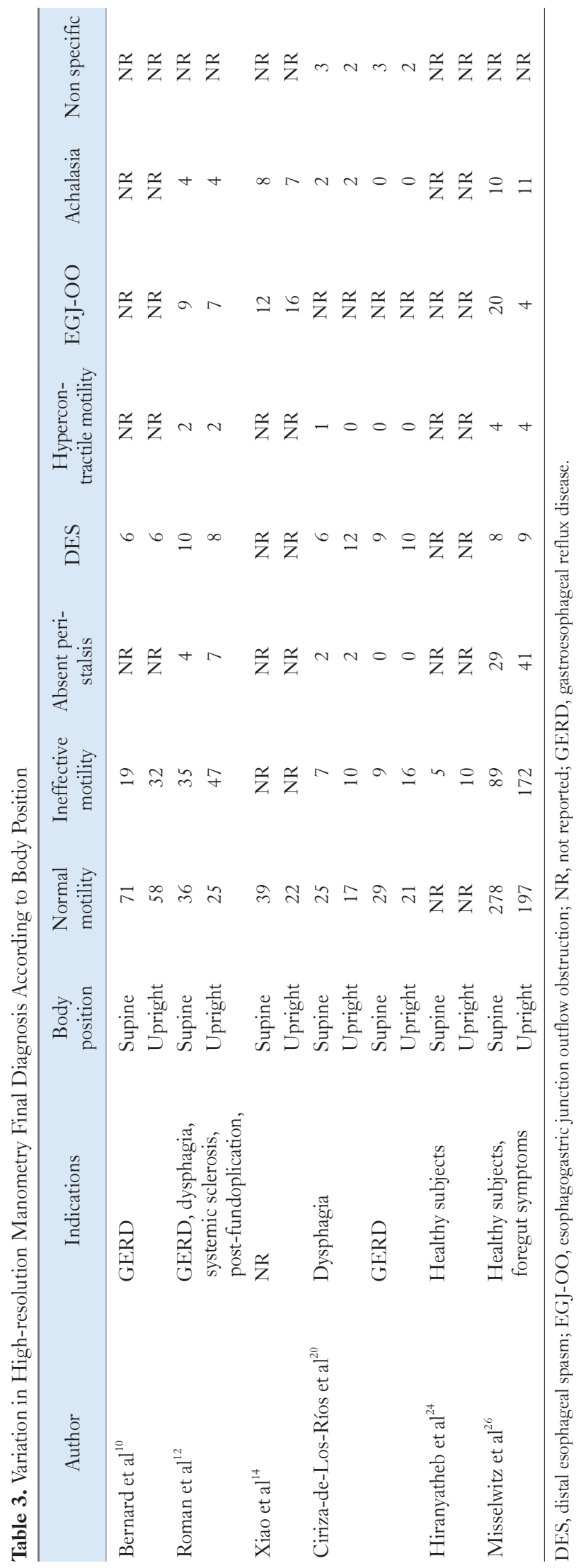

higher DCI in the sitting/upright position.

Overall, the diagnostic agreement in the final manometric diagnosis between the 2 positions varied from $67.6 \%$ to $90.0 \% .^{10,14,26}$ It should be noted that other factors could influence a change in diagnosis in patients undergoing HRM. In fact, reproducibility of HRM may represent the Achille's heel of this technology, and when the test is repeated over time the diagnosis may change. Triadafilopoulos $^{27}$ reported a $41.0 \%$ change in diagnosis in patients who had an initial normal study after a mean interval between studies of 15 months. In contrast, in the only patient with achalasia the diagnosis remained stable over time. This suggests that change in the final diagnosis may not be clinically relevant, and precautions must be taken in the interpretation of HRM findings.

There are some limitations in this study. First, the heterogeneity of subjects included into the analysis: some studies involved both healthy and symptomatic adults. This may introduce a significant bias due to the multiple factors that can affect the HRM results. Second, the studies considered in this review do not assume the same HRM classification or protocol in the assessment of results. Third, no studies considered the most recent HRM tools, such as multiple rapid swallows-DCI ratio and EGJ-contractile integral. ${ }^{28,29}$

Although the results of comparative studies analyzed in the present systematic review are still discordant, the upright/sitting position has more recently emerged as an alternative to the supine position, which appears to be uncomfortable for the patient and probably non-physiological. ${ }^{6}$ However, normal values are needed to establish the most adequate body position for HRM in order to increase the reproducibility of the test. Interestingly, at least 1 clinical study in gastroesophageal reflux disease patients ${ }^{30}$ and one non-comparative study ${ }^{31}$ in normal volunteers considered the semirecumbent position with $30^{\circ}$ sit-back inclination which may be as much as comfortable both for the patient and the physician. Additionally, in this pilot study, the results obtained were similar to those previously described by Pandolfino et $\mathrm{al}^{1}$ and Ghosh et $\mathrm{al}^{2,3}$ in the supine position. More trials should be performed to evaluate if the semi-recumbent or upright position could become the reference standard in the future.

In conclusion, performing HRM in the upright position affect some manometric variables that may change the final manometric diagnosis. Further studies to determine the normal manometric values and evaluate patient reported outcomes and compliance in the sitting and semi-recumbent positions are needed.

Acknowlegements: Work supported by AIRES (Associazione Italiana Ricerca ESofago). 


\section{Financial support: None.}

\section{Conflicts of interest: None.}

Author contributions: Carlo G Riva, Stefano Siboni, Davide Ferrari, and Luigi Bonavina designed the study and wrote the manuscript; and Carlo G Riva, Davide Ferrari, Marco Sozzi, Matteo Capuzzo, Emanuele Asti, and Cristina Ogliari collected the data. All authors reviewed the final version of the manuscript.

\section{References}

1. Pandolfino JE, Ghosh SK, Zhang Q, Jarosz A, Shah N, Kahrilas PJ. Quantifying EGJ morphology and relaxation with high-resolution manometry: a study of 75 asymptomatic volunteers. Am J Physiol Gastrointest Liver Physiol 2006;290:G1033-G1040.

2. Ghosh SK, Pandolfino JE, Zhang Q, Jarosz A, Shah N, Kahrilas PJ. Quantifying esophageal peristalsis with high-resolution manometry: a study of 75 asymptomatic volunteers. Am J Physiol Gastrointest Liver Physiol 2006;290:G988-G997.

3. Ghosh SK, Pandolfino JE, Zhang Q, Jarosz A, Kahrilas PJ. Deglutitive upper esophageal sphincter relaxation: a study of 75 volunteer subjects using solid-state high-resolution manometry. Am J Physiol Gastrointest Liver Physiol 2006;291:G525-G531.

4. Pandolfino JE, Ghosh SK, Rice J, Clarke JO, Kwiatek MA, Kahrilas PJ. Classifying esophageal motility by pressure topography characteristics: a study of 400 patients and 75 controls. Am J Gastroenterol 2008;103:2737.

5. Kahrilas PJ, Bredenoord AJ, Fox M, et al. The Chicago classification of esophageal motility disorders, v3.0. Neurogastroenterol Motil 2015;27:160-174.

6. Herregods TV, Roman S, Kahrilas PJ, Smout AJ, Bredenoord AJ. Normative values in esophageal high-resolution manometry. Neurogastroenterol Motil 2015;27:175-187.

7. Winans CS, Harris LD. Quantitation of lower esophageal sphincter competence. Gastroenterology 1967;52:773-778.

8. Babaei A, Mittal RK. Cardiovascular compression of the esophagus and spread of gastro-esophageal reflux. Neurogastroenterol Motil 2011;23:45-51, e3.

9. Murad MH, Sultan S, Haffar S, Bazerbachi F. Methodological quality and synthesis of case series and case reports. BMJ Evid Based Med 2018;23:60-63.

10. Bernhard A, Pohl D, Fried M, Castell DO, Tutuian R. Influence of bolus consistency and position on esophageal high-resolution manometry findings. Dig Dis Sci 2008;53:1198-1205.

11. Buduhan G, Orlina J, Louie B, Vallieres E, Aye R. Endoscopic and manometric position-related characteristics of the normal gastroesophageal junction. Surg Endosc 2010;24:2165-2169.

12. Roman S, Damon H, Pellissier PE, Mion F. Does body position modify the results of oesophageal high resolution manometry? Neurogastroenterol Motil 2010;22:271-275.
13. Sweis R, Anggiansah A, Wong T, Kaufman E, Obrecht S, Fox M. Normative values and inter-observer agreement for liquid and solid bolus swallows in upright and supine positions as assessed by esophageal highresolution manometry. Neurogastroenterol Motil 2011;23:509-e198.

14. Xiao Y, Nicodème F, Kahrilas PJ, Roman S, Lin Z, Pandolfino JE. Optimizing the swallow protocol of clinical high-resolution esophageal manometry studies. Neurogastroenterol Motil 2012;24:e489-e496.

15. Hoppo T, Komatsu Y, Nieponice A, Schrenker J, Jobe BA. Toward an improved understanding of isolated upright reflux: positional effects on the lower esophageal sphincter in patients with symptoms of gastroesophageal reflux. World J Surg 2012;36:1623-1631.

16. Xiao Y, Read A, Nicodème F, Roman S, Kahrilas PJ, Pandolfino JE. The effect of a sitting vs supine posture on normative esophageal pressure topography metrics and Chicago classification diagnosis of esophageal motility disorders. Neurogastroenterol Motil 2012;24:e509-e516.

17. Zhang X, Xiang X, Tu L, Xie X, Hou X. Esophageal motility in the supine and upright positions for liquid and solid swallows through highresolution manometry. J Neurogastroenterol Motil 2013;19:467-472.

18. Besanko LK, Burgstad CM, Cock C, Heddle R, Fraser A, Fraser RJ. Changes in esophageal and lower esophageal sphincter motility with healthy aging. J Gastrointest Liver Dis 2014;23:243-248.

19. Hashmi S, Rao SS, Summers RW, Schulze K. Esophageal pressure topography, body position, and hiatal hernia. J Clin Gastroenterol 2014;48:224-230.

20. Ciriza-de-Los-Ríos C, Canga-Rodríguez-Valcárcel F, Lora-Pablos D, De-La-Cruz-Bértolo J, Castel-de-Lucas I, Castellano-Tortajada G. How the body position can influence high-resolution manometry results in the study of esophageal dysphagia and gastroesophageal reflux disease. J Neurogastroenterol Motil 2015;21:370-379.

21. Zhang XJ, Xiang XL, Tu L, Xie XP, Hou XH. The effect of position on esophageal structure and function determined with solid-state highresolution manometry. J Dig Dis 2015;16:350-356.

22. Jung KW, Jung HY, Myung SJ, et al. The effect of age on the key parameters in the Chicago classification: a study using high-resolution esophageal manometry in asymptomatic normal individuals. Neurogastroenterol Motil 2015;27:246-257.

23. do Carmo GC, Jafari J, Sifrim D, de Oliveira RB. Normal esophageal pressure topography metrics for data derived from the Sandhill-Unisensor high-resolution manometry assembly in supine and sitting positions. Neurogastroenterol Motil 2015;27:285-292.

24. Hiranyatheb P, Chakkaphak S, Chirnaksorn S, Lekhaka P, Petsrikun K, Somboonpun K. Normal values of high-resolution manometry in supine and upright positions in a thai population. Dig Dis Sci 2018;63:173-183.

25. Pu L, Chavalitdhamrong D, Summerlee RJ, Zhang Q. Effects of posture and swallow volume on esophageal motility morphology and probability of bolus clearance: a study using high-resolution impedance manometry. Gastroenterol Nurs Published Online First: 25 Jun 2019. doi: 10.1097/ SGA.0000000000000356.

26. Misselwitz B, Hollenstein M, Bütikofer S, Ang D, Heinrich H, Fox M. Prospective serial diagnostic study: the effects of position and provocative tests on the diagnosis of oesophageal motility disorders by high-resolution manometry. Aliment Pharmacol Ther 2020;51:706-718. 
27. Triadafilopoulos G, Kamal A, Zikos T, Nguyen L, Clarke JO. Changes in high-resolution manometric diagnosis over time: implications for clinical decision-making. Dis Esophagus 2020;33:doz094.

28. Shaker A, Stoikes N, Drapekin J, Kushnir V, Brunt LM, Gyawali CP. Multiple rapid swallow responses during esophageal high-resolution manometry reflect esophageal body peristaltic reserve. Am J Gastroenterol 2013;108:1706-1712.

29. Nicodème F, Pipa-Muniz M, Khanna K, Kahrilas PJ, Pandolfino JE. Quantifying esophagogastric junction contractility with a novel HRM topographic metric, the EGJ-contractile integral: normative values and preliminary evaluation in PPI non-responders. Neurogastroenterol Motil 2014;26:353-360.

30. Riva CG, Siboni S, Sozzi M, Lazzari V, Asti E, Bonavina L. High-resolution manometry findings after Linx procedure for gastro-oesophageal reflux disease. Neurogastroenterol Motil 2020;32:e13750.

31. Niebisch S, Wilshire CL, Peters JH. Systematic analysis of esophageal pressure topography in high-resolution manometry of 68 normal volunteers. Dis Esophagus 2013;26:651-660. 